\title{
Quaderni
}

QUADERNI Communication, technologies, pouvoir

77 | Hiver 201 1-2012

L'évaluation de la recherche

\section{Les chercheurs et la programmation de la recherche : du discours stratégique à la construction de sens}

Matthieu Hubert, Francis Chateauraynaud et Jean-Michel Fourniau

\section{(2) OpenEdition \\ Journals}

Édition électronique

URL : http://journals.openedition.org/quaderni/556

DOI : 10.4000/quaderni.556

ISSN : 2105-2956

Éditeur

Les éditions de la Maison des sciences de l'Homme

\section{Édition imprimée}

Date de publication : 5 janvier 2012

Pagination : 85-96

Référence électronique

Matthieu Hubert, Francis Chateauraynaud et Jean-Michel Fourniau, «Les chercheurs et la

programmation de la recherche : du discours stratégique à la construction de sens », Quaderni [En ligne], 77 | Hiver 2011-2012, mis en ligne le 05 janvier 2014, consulté le 19 avril 2019. URL : http:// journals.openedition.org/quaderni/556 ; DOI : 10.4000/quaderni.556 


\section{$D$ ossier}

\section{les chercheurs et}

la programmation de la recherche : du discours stratégique à la construction

de sens

Matthieu

Hubert

CONICET

Francis

Chateauraynaud

GSPR EHESS

Jean-Michel Fourniau

IFSTTAR

Centro Ciencia, Tecnologia y Sociedad, Argentine
La place grandissante de la programmation de la recherche s'inscrit dans le cadre de réformes profondes du système de recherche français (Mustar et Larédo, 2002). Elle prend forme dans un ensemble de pratiques (évaluation individuelle et collective, financement sur projets, etc.), de termes (work package, programme blanc, innovation, postdoctorant, deliverable, etc.) et de dispositifs (agences, appels à projets, formes contractuelles de gestion, etc.), qui remettent profondément en question le travail scientifique, touchant non seulement à la pratique quotidienne et au fonctionnement interne des équipes et laboratoires, mais aussi à l'identité professionnelle des chercheurs (Jouvenet, 2011). En particulier, en instituant un certain nombre de règles et de procédures, ces programmes agissent comme des dispositifs contractuels, déléguant une grande partie du travail administratif au monde scientifique, tout en laissant la possibilité à l'agence de programmation de reprendre à tout moment le contrôle sur la marche du projet (Tricoire, 2006). Les scientifiques sont ainsi contrôlés par des experts du « nouveau management » de la recherche, qui favorisent la diffusion d'une conception managériale de la science, susceptible d'affaiblir l'autonomie des professionnels de la recherche (Vilkas, 2009). Leur performance globale est le plus souvent évaluée au moyen de techniques de gestion et d'étalonnage (benchmarking), et valorisée selon la contribution des sciences à la compétitivité économique (Bruno, 2006).

Les réponses des chercheurs aux nouvelles injonctions de performance - leur rejet de telles transformations ou leur engagement dans un nouveau rôle d'entrepreneur scientifique - ont 
souvent été interprétées comme un travail stratégique de démarcation (Boundary work) (Gieryn, 1983), destiné à affirmer et à renforcer les «idéologies professionnelles » de ceux qui différencient ainsi leurs connaissances, leurs compétences et leurs pratiques. En particulier, dans plusieurs travaux (voir par exemple Calvert, 2006, Lam, 2010, Tuunaimen, 2005), le concept de boundary work vise à analyser les discours et les pratiques des chercheurs comme un jeu stratégique destiné à acquérir ressources et prestige, prenant appui sur l'ambiguilté et la flexibilité interprétative des rôles joués, ou des notions, concepts ou catégories employés par les scientifiques, ainsi que sur leur utilisation contingente et ambivalente selon le public ou le contexte d'énonciation. Mobilisant ce concept, Jane Calvert (2006) montre ainsi, par exemple, que la notion de "recherche fondamentale 》 (basic research) a différentes significations selon le contexte dans lequel elle est utilisée - que ce soit au sein de la communauté scientifique d'appartenance ou dans le contexte des agences de financement. Selon cet auteur, une telle utilisation ambivalente permet aux chercheurs de se protéger des demandes d'évaluation ou d'applicabilité des recherches qu'ils souhaitent mener.

Cet article se propose d'apporter quelques éléments de discussion de l'utilisation faite des travaux de Thomas Gieryn (1983), interprétant principalement les réponses des chercheurs en termes stratégiques. En particulier, on montrera qu'une telle approche surestime la rationalité stratégique des chercheurs et l'intelligibilité des transformations qui traversent l'organisation et le pilotage de la recherche scientifique : le foisonnement de dispositifs, d'informations, d'incitations et d'injonctions conduit les cher- cheurs à produire un travail d'interprétation et de construction de sens, qui tend davantage à souligner la diversité persistante des pratiques et des représentations, plutôt que leur adhésion à des principes et des normes promus par les agences de programmation.

Cette analyse repose sur une enquête par entretiens approfondis réalisés en 2010 auprès de quarante-deux chercheurs, enseignants-chercheurs, techniciens, ingénieurs, doctorants, postdoctorants, au sein de dix-huit équipes de recherche en nanosciences et nanotechnologies (nanoS\&T) de la région parisienne ${ }^{1}$. Les nanoS\&T se caractérisent notamment par la multiplication de dispositifs de politique scientifique qui en structurent l'organisation, ce qui fait de ce domaine un terrain d'étude significatif des transformations de la politique de recherche française. En particulier, plus que d'autres domaines, ce champ de recherche est traversé par de multiples injonctions de performance, qui portent aussi bien sur les procédures (construction des projets selon des formats prédéterminés, rédaction de rapports d'activités, recherche d'informations concernant les programmes existants et leurs modalités de fonctionnement, etc.) que sur les résultats attendus (l'appartenance au champ des nanoS\&T, la mise en avant de collaborations interdisciplinaires, les possibilités d'applications ou de brevets, les relations avec des partenaires industriels, etc.). De ce fait, les nanoS\&T apparaissent comme particulièrement pertinentes pour interroger la performativité des injonctions gestionnaires, ainsi que le caractère plus ou moins prescriptif de ces nouveaux dispositifs.

L'article s'organise en deux temps. La première partie décrit les stratégies déployées par les cher- 
cheurs pour prendre en compte les injonctions de performance qui accompagnent la politique de soutien aux nanoS\&T. La deuxième partie analyse les arguments critiques permettant de saisir comment les chercheurs font sens de la politique d'intensification de la programmation.

\section{Interpréter - et jouer avec - les exigences de la programmation}

Depuis la fin des années 1990, les nanoS\&T sont inscrites dans les priorités de politique scientifique de la plupart des pays industrialisés et émergents (Laurent, 2010). En France, le domaine des nanoS\&T a été façonné par une volonté de politique scientifique formulée à l'échelle nationale, dont le pilotage et la mise en œuvre se sont progressivement renforcés au moyen d'une multitude de dispositifs ${ }^{2}$. Malgré leur variété, tant du point de vue de leurs modalités d'organisation que de leurs critères d'évaluation et de leurs finalités, ces programmes mettent pour la plupart l'accent sur la recherche finalisée et l'innovation technologique, finançant notamment des projets de recherche partenariale entre laboratoires académiques et industriels. Beaucoup visent également à structurer la recherche sur une base territoriale, et à rassembler les acteurs selon leur proximité géographique.

Dans leurs réponses, les chercheurs interrogés prennent position vis-à-vis des injonctions de la programmation en nanoS\&T, leur bien-fondé, leurs apports, leurs limites et leurs éventuels effets pervers. Selon certains, ce foisonnement de dispositifs profite du flou et de la flexibilité entourant le «mot-clef nano » (E1): au niveau des politiques scientifiques, "il y a un emploi volontaire du terme nanotechnologie et un jeu sur nanotechnologie/nanoscience »(E5). Mais quels rapports les chercheurs en nanoS\&T entretiennent-ils concrètement avec cette politique scientifique? On peut tenter d'analyser les réponses des chercheurs à cette question en distinguant deux niveaux : d'abord, quelles nouvelles activités ces dispositifs génèrent-ils dans la vie quotidienne du laboratoire ? Ensuite, comment les chercheurs intègrent-ils les nouvelles injonctions de performance dans leurs stratégies de recherche?

\section{La critique de l'exigence de "rendre des comptes sur tout "}

Pour nombre de chercheurs interrogés, la programmation de la recherche marque une évolution importante du métier de chercheur, qui s'observe d'abord au travers des nouvelles compétences administratives, managériales et relationnelles demandées aux chercheurs (Jouvenet, 2011, Barrier, 2011). La transformation du métier est notamment constatée au travers de l'inflation des tâches administratives, destinées à " rendre des comptes sur tout »(E27). Ces nouvelles exigences administratives, souvent analysées en terme d'accountability (Neyland, 2007), et supposées rendre les organisations transparentes et responsables, pèsent sur les activités quotidiennes des chercheurs, puisque les chercheurs en poste y consacrent une partie croissante de leur temps de travail - un temps qu'une chercheuse évalue à un tiers de son temps de travail total.

Parmi ces activités administratives de gestion, les chercheurs distinguent les pratiques stratégiques, qui sont au cœur de leur activité de recherche, de celles qui sont plus périphériques, relevant 
davantage de la routine bureaucratique (Dahan et Mangematin, 2010). Les premières englobent notamment la recherche d'informations concernant les programmes, leurs critères d'évaluation et les chances de les obtenir, le travail pour constituer des réseaux ou des "consortiums », ainsi que l'ensemble du travail de mise en forme des projets scientifiques destinés à être évalués par des pairs. Les pratiques jugées plus périphériques sont notamment celles destinées à justifier des dépenses réalisées, à mobiliser les partenaires pour répondre aux rapports d'avancements des projets (des "delivrables », dans le jargon du management de la recherche), et à rendre compte des activités réalisées selon les formats préétablis par les agences de financement.

Beaucoup des chercheurs interrogés critiquent l'inflation des tâches liées à ces activités administratives non stratégiques, envahissant ainsi le temps disponible pour d'autres activités plus valorisées ou considérées comme plus stratégiques : "Ça ne fait pas partie de mon travail que d'aller faire des rapports finaux tous les six mois ou tous les dix-huit mois, ou d'aller présenter mes résultats à Bruxelles devant un commissaire européen » (E28). L'exigence de « rendre des comptes sur tout » (E27) est ainsi critiquée, parce qu'elle ne rentre pas dans le périmètre des activités scientifiques jugées comme faisant véritablement partie du métier de chercheur. Elle induit une charge de travail qui n'est plus disponible pour d'autres activités considérées comme étant plus stratégiques.

Malgré les critiques évoquées, la plupart des chercheurs prennent néanmoins en compte les injonctions de performance de la programmation.
Ils soulignent que les pressions politiques pour une plus grande utilité sociale ont réellement modifié la façon dont les scientifiques perçoivent et présentent leur activité de recherche, en insistant sur l'importance des applications potentielles et de l'implication des industriels dans les projets collectifs. Mais la prise en compte des exigences de la programmation n'est pas suivie des mêmes effets selon les injonctions concernées et les contextes de travail dans lesquels évoluent les chercheurs.

\section{Les jeux stratégiques pour prendre en compte les injonctions de performance}

La prise en compte des exigences d'accountability fait l'objet d'un véritable jeu stratégique des chercheurs. Par exemple, ceux-ci reconnaissent volontiers que les applications envisagées sont souvent bien plus lointaines et incertaines que ne le laisse supposer la justification d'une demande de financement. Par ailleurs, les chercheurs disent découper leurs projets (ou décliner différentes versions du même projet) pour le soumettre à différents programmes, profitant de la variété des attendus d'un programme à l'autre : " le modèle de recherche pourrait devenir une contrainte. Aujourd'hui, ça n'en est pas une car on arrive à contourner le système et à faire en sorte de taper dans plusieurs appels d'offre sans modifier la finalité de ce qu'on a envie de développer, mais ça pourrait le devenir » (E1). Plus généralement, les pratiques d'intéressement (d'un évaluateur ou d'un partenaire) et de justification (des activités réalisées ou à réaliser) sont souvent considérées par les chercheurs comme étant éloignées des réalités du laboratoire : "ça ne correspond pas toujours au vrai travail de recherche. Donc il 
y a un décalage entre le discours et ce qu'on fait réellement " (E14). En d'autres termes, le compte-rendu des activités scientifiques ne peut que difficilement correspondre à une description réaliste de la vie du laboratoire.

Toutefois, la mise en œuvre de ces stratégies se heurte à un certain nombre de difficultés pratiques que les chercheurs se doivent alors de résoudre. On peut distinguer au moins deux modalités d'adaptation à ces contraintes.

D'une part, pour répondre aux finalités redéfinies par les dispositifs de programmation, les chercheurs doivent apprendre de nouveaux savoir-faire, comme la capacité à intéresser des partenaires industriels, ou la connaissance des procédures et l'expérience pratique du dépôt de brevets. Cet apprentissage ne va pas de soi : «on se dit qu'il faudrait commencer à penser en ces termes, penser à des projets pour pouvoir breveter (mais) on n'a pas de culture du brevet; on ne sait pas ce qui est brevetable ou pas » (E40). Ainsi, plus généralement, la prise en compte des injonctions de performance ne s'accompagne-telle pas toujours des conditions d'accompagnement, matérielles ou institutionnelles, permettant aux chercheurs de réaliser les nouveaux objectifs demandés ${ }^{3}$.

D'autre part, il s'agit aussi d'inscrire le projet scientifique dans un format préétabli. Ainsi, par exemple, le fait de devoir expliciter " noir sur blanc » des applications potentielles ou des « $v i$ sées applicatives » pour augmenter ses chances d'obtention d'un financement peut s'apparenter à une véritable " gymnastique intellectuelle»: " en fait, ce qui est curieux, c'est que ça ne nous amène pas forcément à travailler vers des objectifs plus appliqués. Ça nous oblige à une certaine gymnastique... intellectuelle. Mais c'est vrai que là il y a un changement important, ces dernières années... On est un peu victime de ça. C'est-à-dire quand on définit notre recherche, très souvent, mais surtout les jeunes chercheurs d'ailleurs, j'ai remarqué, ils définissent leur recherche par rapport à des applications " (E14). L'exercice argumentatif consiste alors à respecter les contraintes de l'appel d'offre, tout en contournant ses finalités pour qu'il fonctionne de manière plus souple.

Finalement, si les chercheurs en nanoS\&T déploient, comme on l'a vu, des stratégies de conformité, d'évitement ou de contournement, afin de prendre en compte les nouvelles injonctions de la programmation, ils n'adhèrent pas pour autant complètement aux valeurs et aux normes promues par les agences de programmation. Les comportements stratégiques des chercheurs ne signifient pas leur adhésion aux politiques scientifiques qui les sous-tendent. Ainsi, l'explication stratégique, dont l'unique objet serait de favoriser une évaluation positive des agences de financement, évacue-t-elle d'autres formes d'interprétations des comportements et des discours. C'est ce que nous allons détailler dans la suite.

\section{Mettre à l'épreuve le sens de la programmation}

Afin de dépasser la lecture stratégique du discours des chercheurs, il est utile d'analyser les arguments critiques qu'ils formulent à son encontre. Au moins deux types de critiques se dégagent des entretiens : le premier type d'argument, plus mar- 
qué idéologiquement, oppose deux visions de la science supposées inconciliables; le second type de réponse, plus pragmatique, pointe certaines contradictions et ambivalences véhiculées par les injonctions de performance. L'analyse de ces arguments permet de saisir comment les chercheurs font sens de la politique scientifique qui repose sur une intensification de la programmation.

\section{L'alternative entre "science pour l'argent" et "science pour le savoir"}

Pour les chercheurs, l'interprétation du sens profond de la programmation n'a rien d'évident. Pourtant, les usages stratégiques de la variété des programmes existants nécessitent une réelle compréhension des attentes des politiques scientifiques à l'œuvre. Saisir le rôle que les décideurs et les financeurs souhaitent faire jouer à la recherche est même souvent un préalable à la formulation et l'exécution des stratégies précédemment évoquées de conformité, d'évitement ou de contournement.

La compréhension du sens plus général du nouveau modèle de recherche que véhicule la programmation peut passer par une critique radicale de celle-ci. Par exemple, elle peut s'exprimer au travers d'une dénonciation de la mise sous tutelle par le politique : " L'ANR a été créé par l'État pour contrôler la recherche. Peut-être pour la rendre meilleure. Moi je ne suis pas sûr »(E6). L'intensification de la programmation peut aussi être considérée comme une volonté délibérée des pouvoirs publics d'affaiblir les recherches les plus «fondamentales » : «Oui, oui, je crois que les gens qui font du fondamental, ils se sentent vraiment menacés. Ça se réduit, je n'ai pas de chiffre, mais il y a une telle volonté, une telle demande de rendre des comptes sur tout ; il y a quand même des domaines où vous avez du mal à rendre des comptes parce que ce n'est pas chiffrable » (E27).

Une formulation alternative de la cohérence générale et du sens global de la politique scientifique de programmation fait intervenir la référence aux valeurs associées à la science, et aux normes censées organiser la communauté scientifique (Merton, 1973). Le renforcement de la programmation est alors interprété comme un choix politique devant l'alternative entre « la science pour le savoir » et la science " pour faire de l'argent » (E9); ou, dans une version proche, entre le bien public et l'appropriation privée : " Peut-être que j'ai une vision un peu idéaliste de la science et de la recherche fondamentale. Moi, je pars du principe que du moment où l'on fait de la recherche au niveau fondamental, bien qu'on envisage sérieusement des applications, qu'elles soient à long terme ou à court terme, il faut un certain partage des connaissances, ce qu'exclut un brevet. Un brevet, je le vois plus comme un impératif pour certaines personnes qui s'intéressent à certains aspects financiers ou économiques, mais je pense que, du moins dans l'état actuel d'avancement des nanosciences, pour faire évoluer les choses, on n'a pas besoin de brevet » (E15).

Dans cette perspective, les chercheurs redoutent que la programmation transforme, de manière plus durable, le sens qu'ils donnent à leur engagement professionnel, ce que signifie faire de la science, ainsi que les valeurs et les finalités qui y sont associées. Le choix politique de la programmation 
traduit alors la volonté de désigner des gagnants et des perdants, d'opposer ceux qui agiraient selon une rationalité en finalité (la figure de l'entrepreneur scientifique, fin stratège, produisant une recherche orientée par le contexte d'application), à ceux dont les prises de position relèveraient d'une rationalité en valeur (la figure de l'académique, produisant des connaissances partagées et évaluées par sa communauté de recherche).

\section{Les incertitudes, les contradictions et les ambivalences de la programmation}

D'autres réponses - pouvant parfois pourtant venir des mêmes chercheurs - font une critique plus ciblée de la programmation. Elles pointent certaines incertitudes et ambivalences véhiculées par les injonctions de performance. Elles se demandent notamment si les conditions sont réunies pour pouvoir répondre aux attentes de la programmation. En effet, d'une part, le foisonnement de dispositifs, la multiplication des objectifs et la diversification des tâches à accomplir mettent les chercheurs en situation d'incertitude quant à leurs possibilités et leurs capacités de répondre aux injonctions : " Mais on ne peut pas nous demander de tout faire non plus. C'est ça le gros problème, c'est qu'on nous demande de tout faire : d'enseigner, de faire de l'évaluation, d'inventer en sciences, diriger un labo, déposer des brevets [rires]... en plus ce n'est pas la même chose : comme on fait tout le temps des publications, on sait comment faire, mais un brevet, j'imagine, les premières fois, il y a beaucoup de travail à faire » (E34).

D'autre part, au-delà des incertitudes, certains des chercheurs interrogés dénoncent les injonc- tions contradictoires portées par les différents dispositifs de programmation. En particulier, ils sont tiraillés entre les incitations à l'innovation et les mécanismes même de la programmation et de l'évaluation. Ainsi, les recherches plus exploratoires ou plus risquées - les activités de "défrichage ", comme les nomme une chercheuse (E17) -, destinées à évaluer le potentiel d'un champ de recherche peu exploré jusqu'alors, sont jugées comme étant insuffisamment financées par les politiques de soutien aux nanoS\&T. Ainsi, symptôme de cette frilosité qui serait encouragée par la place accrue prise par la programmation, certaines équipes disent ne présenter que des projets déjà engagés, pour lesquels ils peuvent déjà faire la preuve de résultats intéressants. Plus généralement, ce sont les justifications en amont des recherches - notamment le fait de devoir prévoir a priori toutes les dépenses qui vont être engagées durant le projet - qui sont ressenties comme contradictoires avec les contraintes de l'activité de recherche, puisque celle-ci est considérée comme étant par nature incertaine.

D'autres effets pervers sont associés aux incitations que produiraient les dispositifs de programmation. En particulier, les appels à projets thématiques sont parfois vus comme des incitations à l'opportunisme, décourageant notamment la poursuite d'un projet scientifique continu, cumulatif et suivi sur une longue période de temps, au profit de bifurcations stratégiques, pour lesquelles les intérêts individuels et collectifs ne sont pas toujours convergents au sein d'une même équipe : "Ça fait qu'au niveau du laboratoire ou d'une équipe, on n'arrête pas de faire des bifurcations. Ça peut être un enrichissement mais ça peut avoir pour consé- 
quence que vous n'approfondissez pas un sujet. Moi, $j$ 'ai tendance à dire que le cadre de trois ans est trop court. Il faudrait pouvoir enchaîner au moins six ans sur un même sujet » (E27). Le court terme serait alors privilégié, au détriment $\mathrm{du}$ long terme (ou, selon les arguments, sans tenir compte de la durée réelle des projets, qui ne cadre pas nécessairement avec les limites temporelles fixées a priori et sans distinction de projet par les agences). Au-delà de l'horizon temporel, ce sont les divergences de " tempo » (ou de " calendriers ») qui posent des problèmes organisationnels et stratégiques accrus, lorsqu'il s'agit d'aligner les délais et les objectifs d'un projet scientifique individuel (celui d'un doctorant et ses impératifs de reconnaissance académique, par exemple) et ceux d'un projet scientifique collectif ayant obtenu un financement (regroupant une ou plusieurs équipes).

D'autres contradictions sont également liées aux divergences entre le système d'évaluation des chercheurs (et des équipes) et le système d'évaluation des projets, qui valorisent différemment des critères comme l'interdisciplinarité, le dépôt de brevets ou la coopération industrielle. Publier et breveter sur un même projet pose aussi des problèmes, l'un retardant l'autre, voire le rendant impossible. Certains chercheurs dénoncent alors les injonctions contradictoires qui promeuvent conjointement le partage et le secret : " cette dissociation entre 'il faut ne rien dire pour pouvoir breveter' et 'il faut publier dans des super journaux pour valoriser', c'est un petit peu contradictoire » (E40) ${ }^{4}$.

Dans ce dernier cas, en faisant référence à la norme du partage des connaissances produites, il s'agit donc moins de s'opposer frontalement à toute transformation du métier de chercheur et du système de recherche, que de souligner la perte de sens lié au foisonnement de dispositifs et de critères d'évaluation des projets, leurs ambiguités et leurs contradictions. En mettant en avant les incertitudes et les ambivalences de la programmation, ces discours relativisent la distinction précédemment évoquée entre des chercheurs qui agiraient selon une rationalité en finalité ("la science pour l'argent »), et ceux dont les prises de position relèveraient d'une rationalité en valeur ( la science pour le savoir »). Au regard d'injonctions de performance dont le sens plus profond est difficile à percevoir pour les chercheurs eux-mêmes, les politiques scientifiques peuvent difficilement obtenir l'adhésion des chercheurs - et ce, que ceux-ci raisonnent en valeur ou en finalité. En refusant de co-construire, avec les chercheurs, le sens et les finalités des dispositifs d'organisation de la recherche, elles s'exposent davantage, on l'a vu, au contournement, voire au rejet pur et simple.

\section{Conclusion}

Les réponses des chercheurs aux nouvelles injonctions de performance, leur rejet des transformations de la recherche ou leur engagement dans un nouveau rôle d'entrepreneur scientifique, ont souvent été interprétés comme un travail stratégique de démarcation (boundary work) (Gieryn, 1983). En faisant ressortir la diversité des formes d'expression et d'argumentation que les chercheurs déploient à propos de la prise en compte des injonctions de la programmation, allant des figures de dénonciation des logiques mercantiles ou utilitaristes, aux marques de résignation vis- 
à-vis de l'inflation des tâches bureaucratiques visant à rendre compte de l'activité scientifique, en passant par des stratégies alternatives d'adaptation, d'alignement et de contournement des dispositifs de politique scientifique, l'analyse des discours de chercheurs montre qu'une telle approche surestime la rationalité stratégique des chercheurs et l'intelligibilité des transformations qui traversent la recherche scientifique.

En particulier, les discours recueillis rendent bien compte de l'incertitude et de la confusion que rencontrent les chercheurs au regard de la complexité et $\mathrm{du}$ foisonnement de dispositifs de politique scientifique ayant accompagné la première décennie de politiques de soutien aux nanoS\&T. Ainsi, les chercheurs interrogés, bien que directement concernés par la thématique, ne connaissentils pas l'ensemble de ces dispositifs et ils n'en cernent pas nécessairement tous les enjeux, ni même le fonctionnement, qui leur semblent parfois opaque ou contradictoire.

La confusion et les ambivalences de la programmation conduisent les chercheurs à produire un travail d'interprétation et de construction de sens, qui peut être analysé en termes de sensemaking (Weick et al., 2005) - un concept qui permet d'éclairer la recherche et la formulation de repères destinés à faire sens des nouveaux horizons normatifs qui se trouvent ainsi déployés par la programmation en nanoS\&T. L'utilisation de ce concept permet alors de souligner que 1'explication stratégique du discours des chercheurs, véhiculée par l'utilisation faite du concept de boundary work, évacue d'autres formes d'interprétations et de constructions argumentatives, où le sens que les chercheurs donnent à leur engagement professionnel et à la programmation de la recherche est en jeu.

L'analyse faite ici montre que cette construction de sens peut s'exprimer de différentes manières. Elle peut non seulement être formulée au travers d'une critique radicale de la programmation, qui est alors interprétée comme un choix politique devant l'alternative entre « la science pour le savoir » et « la science pour l'argent 》 - ce discours de démarcation étant volontairement déconstruit par les approches en termes de boundary work. Mais elle peut aussi s'exprimer au travers d'une critique plus pragmatique des ambivalences et des contradictions véhiculées par les différents dispositifs de programmation, prenant moins pour cible la valeur de performance ou la norme d'utilité sociale, que la cohérence d'ensemble du projet politique et l'efficacité de sa mise en œuvre - ces derniers arguments étant insuffisamment saisis par les approches qui se focalisent uniquement sur la dimension stratégique du discours des chercheurs.

Cette recherche a bénéficié du soutien financier du programme ANR RTB +003 . 


\section{$R \cdot E ́ F \cdot \dot{E} \cdot R \cdot E \cdot N \cdot C \cdot E \cdot S$}

J. BARRIER, « La science en projets : financements sur projet, autonomie professionnelle et transformations du travail des chercheurs académiques », Sociologie du travail, n53, 2011, pp. 515-536.

I. BRUNO, Déchiffrer "l'Europe compétitive ». Étude du benchmarking comme technique de coordination intergouvernementale dans le cadre de la stratégie de Lisbonne, Paris, Thèse de doctorat de l'IEP de Paris, 2006.

J. Calvert, "What's special about basic research?", Science, Technology \& Human Values, vol.31, n², 2006, pp. 199-220.

A. DAHAN, V. MANGEMATIN, « Recherche ou temps perdu ? Vers une intégration des tâches administratives au métier d'enseignant-chercheur ", Gérer et Comprendre, Annales des Mines, $n^{\circ} 102$, 2010, pp. 14-24.

J. EVANS, "Industry collaboration, scientific sharing, and the dissemination of knowledge", Social Studies of Science, vol.40, n5, 2010, pp. 757-791.

T. GIERYN, "Boundary-Work and the Demarcation of Science from Non-Science: Strains and Interests in Professional Ideologies of Scientists", American Sociological Review, vol.48, n6, 1983, pp. 781-795

M. JOUVENET, «Profession scientifique et instruments politiques : l'impact du financement « sur projet » dans les laboratoires de nanosciences », Sociologie du travail, n53, 2011, pp. 234-252.

A. LAM, "From 'Ivory Tower Traditionalists' to 'Entrepreneurial Scientists'? Academic Scientists in Fuzzy University - Industry Boundaries",
Social Studies of Science, vol.40, n², 2010, pp. 307-340.

B. LAURENT, Les politiques des nanotechnologies. Pour un traitement démocratique d'une science émergente, Paris, Éditions Charles Léopold Mayer, 2010.

R. MERTON, The sociology of science. Theoretical and empirical investigations, Chicago \& London, The University of Chicago Press, 1973. P. MUSTAR, P. LARÉDO, "Innovation and research policy in France (1980-2000) or the disappearance of the Colbertist state", Research policy, $\mathrm{n}^{\circ} 31,2002$, pp. 55-72.

D. NEYLAND, "Achieving transparency: the visible, invisible and divisible in academic accountability networks", Organization, vol.14, n'4, 2007, pp. 499-516.

A. TRICOIRE, « Externaliser la contrainte. Le dispositif de pilotage d'un projet de recherche communautaire ", Terrains \& Travaux, ${ }^{\circ} 11$, 2006, pp. 122-139.

J. TUUNAINEN, "Contesting an hybrid firm at a traditional university”, Social Studies of Science, vol.35, n², 2005, pp. 173-210.

C. VILKAS, « Des pairs aux experts : l'émergence d'un 'nouveau management' de la recherche scientifique? » Cahiers Internationaux de Sociologie, n¹26, 2009.

K. WEICK, K. SUTCLIFFE, D. OBSTFELD, "Organizing and the process of sensemaking", Organisation science, vol.16, $\mathrm{n}^{\circ} 4,2005$, pp. 409-421. 


\section{$\mathrm{N} \cdot \mathrm{O} \cdot \mathrm{T} \cdot \mathrm{E} \cdot \mathrm{S} \mathrm{R} \cdot \mathrm{E} \cdot \mathrm{S} \cdot \mathrm{U} \cdot \mathrm{M} \cdot \dot{E}$}

1. Dans la suite, on utilisera le terme générique de « chercheur » pour les désigner indifféremment.

2. Citons, parmi les dispositifs participant du soutien à la recherche en nanoS\&T : le Réseau des Micro et Nanotechnologies dès 1999, puis le réseau des grandes centrales technologiques Renatech à partir de 2003. Ensuite, dans la seconde moitié de la décennie : le programme P-Nano de 1>ANR, cinq des « pôles de compétitivité » existants, trois des réseaux Thématiques de Recherche Avancée (RTRA), cinq centres de transfert de technologie labellisés « Instituts Carnot », une dizaine d'EQUIPEX (« équipements d'excellence »), ainsi qu'une dizaine de LABEX (« laboratoires d'excellence ») issus du premier appel à projets de 2010 .

3. Sur ce point, les différences entre institutions sont relativement tranchées, entre l'université, le CNRS et le CEA.

4. Il faudrait évidemment entrer davantage dans les détails de l'argumentation des chercheurs car, dans les discours, ce n'est pas tant que le partage soit associé au monde scientifique, et le secret au monde industriel, mais plutôt que la tension et la relation entre partage et secret sont perçues et gérées différemment par les mondes scientifiques et industriels (cf. Evans, 2010).
Les réponses des chercheurs aux nouvelles injonctions de performance ont souvent été interprétées comme un discours stratégique de démarcation (boundary work). Les auteurs montrent qu'une telle approche surestime la rationalité stratégique des chercheurs et l'intelligibilité des transformations qui traversent la recherche scientifique. À partir d'une analyse des discours de chercheurs sur les programmes français de recherche en nanosciences et nanotechnologies, ils montrent que le foisonnement de dispositifs, d'informations et d'incitations conduit les chercheurs à produire un travail d'interprétation et de construction de sens, qui tend davantage à souligner la diversité persistante des pratiques et des représentations, plutôt que l'adhésion des chercheurs à des principes et des normes promus par la programmation. Cette construction du sens peut non seulement s'exprimer au travers d'une critique radicale de la politique scientifique menée, mais aussi via une critique plus pragmatique des ambivalences et des contradictions véhiculées par les différents dispositifs de programmation.

\footnotetext{
Abstract

Researchers' answers to the new performance imperatives have been interpreted in terms of strategic boundary work. The authors show that such an approach overestimates the strategic rationality of researchers and the intelligibility of the transformations in the scientific field. The paper analyses the discourses of nanoscience and nanotechnology researchers about French science policy. It shows that the proliferation of devices, information and incentive leads researchers to interpret, and make sense of, the new context. The analysis of this interpretative work underlines the enduring diversity of practices and representations,
} 
rather than an adherence to the principles and norms of science policy. Such a sense-making does not only manifest itself through a radical criticism of science policy, but also through a more pragmatic criticism of its ambivalence and contradictions. 